\title{
Spark Ignition of Propane-Air Mixtures Near the Minimum Ignition Energy: Part I. An Experimental Study
}

\author{
Y. KO \\ Department of Mechanical Engineering and Applied Mechanics, University of Michigan, Ann Arbor, MI \\ 48109
}

R. W. ANDERSON

Engine Research Dept., Research Staff, Ford Motor Co., Dearborn, MI 48121-2053

and

\begin{abstract}
V.S. ARPACI
Department of Mechanical Engineering and Applied Mechanics, University of Michigan, Ann Arbor, MI 48109
\end{abstract}

\begin{abstract}
Kernel growth from a spark in propane-air mixtures at atmospheric pressure is studied in a constant volume bomb with a high-speed laser schlieren system. The spark current and vpltage waveforms of an inductive ignition source are simultaneously recorded with the photographic recordings. The temporal growth of the measured equivalent radii at conditions near the minimum ignition energy shows the existence of a critical radius and the influence of the critical radius on kernel development. In addition, it is shown that the net spark power for ignition can be estimated using data from minimum ignition energy, electrode fall energy losses, and spark calorimetry experiments. These results are used in Part II to develop a model for kernel growth.
\end{abstract}

\section{NOMENCLATURE}

A

$C_{p}$

$D$

d

$D_{A}$

$E_{d}$

$E_{f}$

$E_{G}\left(\dot{E}_{G}\right)$ gion fuel or limiting reactant constant pressure specific heat electrode diameter gap size binary diffusion coefficient of $A$ into mixture measured spark discharge energy discharge energy into the fall respark energy (power) that remains in the gas

$E_{m}$
$E_{p}\left(\dot{E}_{p}\right)$
$I_{s}$
$k$
$\mathrm{Le}_{A}$
$r$
$r_{c}$
$r_{\text {eq }}$
$T$
$t$
$t_{d}$

minimum ignition energy for a given condition discharge energy (power) into the positive column measured secondary current thermal conductivity Lewis number $\left(=k / \rho C_{p} D_{A}\right)$ radius or spatial coordinate critical radius equivalent kernel radius temperature time or time coordinate discharge duration

Copyright (C) 1991 by The Combustion Institute Published by Elsevier Science Publishing Co., Inc. 


$\begin{array}{ll}t_{0} & \text { time constant for } \eta_{d}(t) \\ U & \text { laminar burning velocity } \\ V & \text { volume of the kernel } \\ V_{(d)(a, f)(c, f)} & \begin{array}{l}\text { (measured discharge) (anode fall) } \\ \text { (cathode fall) voltage }\end{array}\end{array}$

\section{Greek Symbols}

$\begin{array}{ll}\eta_{d}(t) & \text { instantaneous discharge efficiency } \\ \eta_{d}\left(t_{d}\right) & \text { average discharge efficiency over } \\ & \text { discharge duration } t_{d} \\ \eta_{d}^{*} & E_{G} / E_{p} \text { in Table } 4 \\ \eta_{g} & \text { discharge efficiency due to quench- } \\ & \text { ing } \\ \rho & \text { density } \\ \phi & \text { equivalence ratio }\end{array}$

\section{Special Symbols}

$\begin{array}{ll}\mathscr{E} & \text { activation energy } \\ \Re & \text { universal gas constant }\end{array}$

\section{Subscripts}

$\begin{array}{ll}a & \text { adiabatic flame } \\ b & \text { burned gas of steady spherical } \\ & \text { flame } \\ u & \text { unburned fresh mixture }\end{array}$

\section{INTRODUCTION}

IC engine operation at fuel-lean or highly dilute EGR conditions for better fuel economy and emissions has received increased attention. The lean operating limit at these conditions needs to be extended to resolve problems such as reduced drivability and increased hydrocarbon emissions. These problems can result from slow initial flame kernel development, leading to slow burning cycles, failure of flame initiation, or incomplete flame propagation [1]. Efforts to extend the lean operating limit require an improved understanding of flame initiation through flame kernel development. A better understanding of flame initiation is, in turn, closely related to that of flame kernel development at conditions near the minimum ignition energy. This knowledge helps to understand the slow burn, misfire, and partial burn cycles observed in fuel-lean operation. Following this increased attention to the fundamental aspects of spark ignition, the effect of numerous factors on ignition is currently qualitatively understood [2-7]. Quantitative prediction of ignition enhancement, however, continues to demand further research on spark initiation for even well-defined quiescent mixtures.

Because of the size of the literature, no attempt is made here for a complete review (see, for example, Lim [8] and Ko [9]). There are valuable data in the literature not only on the minimum spark ignition energy itself but also on the effect of many parameters on the minimum spark ignition energy in quiescent or flowing mixtures $[2-4$, 10-25]. The parameters of interest include electrical breakdown and discharge characteristics, electrode characteristics, pressure, temperature, and flow characteristics, and mixture characteristics. Accurate information on the minimum ignition energy is essential in the development of spark ignition theory and in the design of ignition systems as well as in safety considerations.

According to Refs. 2-4 and 12-14, the minimum ignition energy decreases with increasing gap size until the gap size reaches the critical value, which is called the quenching distance. Beyond the quenching distance, the minimum ignition energy remains nearly constant over a considerable range of the gap size and then increases linearly with further increases of the gap dimension. The minimum ignition energy also varies with the discharge duration and there exists an optimum spark duration that depends on the mixture strength and quenching effect of the electrodes $[4,10,17-19]$. The absolute minimum ignition energy is, therefore, the minimum ignition energy at a gap size of quenching distance with an optimal spark duration (i.e., minimum of minimum ignition energy). Accurate data not only on the quenching distance but also on the optimal duration are, therefore, important in determining the absolute minimum ignition energy.

Data on the minimum ignition energy with an inductive ignition source show a transition band in the spark energy level between ignition and nonignition for a given condition (see, for example, Refs. 21 and 22). The ignition probability 
with the energy level below the lower limit is $0 \%$ whereas the probability with the energy level above the upper limit is $100 \%$. The ignition probability increases as the energy level increases between the two limits. The occurrence of either ignition or nonignition for a given spark energy level within the transition band is a consequence of perturbations in the breakdown energy, the subsequent discharge, and the location of the spark channel and flame kernel [i.e., the subsequent discharge has less energy and shorter duration when the breakdown voltage (energy) is high and conversely when low]. These perturbations are inherent with an inductive spark ignition system because of the statistical nature of the electrical breakdown at the gap. The effect of these perturbations becomes less significant as the energy level approaches either of the limits, leading to ignition or nonignition. The spark energy level with $50 \%$ ignition probability is usually-defined as the minimum ignition energy for a given condition.

The minimum ignition energy in the literature is used to denote either the spark energy level with $50 \%$ ignition probability for a given condition or the absolute minimum ignition energy. The minimum ignition energy in the present study denotes the minimum ignition energy with the former definition. Some practical spark ignition systems have electrodes with a gap size smaller than the quenching distance and discharge durations different than the optimal value. Therefore, data on the minimum ignition energy for a given condition in addition to the absolute minimum energy values are also useful in practice.

Data on flame kernel growth, when combined with minimum ignition energy data, provide supplemental information for a better understanding of spark ignition. The flame kernel, measured to be spherical $[8,26]$, is numerically shown to provide the best predictions of experimentally observed phenomena in spark ignition $[27,28]$. Observation of a developing spark kernel also reveals the existence of a critical radius $r_{c}$ that the flame kernel must reach for successful development [9, 29-33]. Recent theoretical studies on spherical flame initiation [33-35] explicitly show the existence of this critical radius and explain its role in flame kernel development. The importance of diffusion in spherical flame initiation is also well demonstrated and successful initiation is found to require that the spark energy and power input be sufficient to drive the flame kernel beyond the critical radius. Using an asymptotic analysis, Champion et al. [33] find the critical radius $r_{c}$ to be

$$
\begin{aligned}
r_{c}= & \frac{(k)_{T_{a}}}{\left(C_{p}\right) T_{a}(\rho) T_{u} U_{a}} \frac{T_{a}}{T_{b} \mathrm{Le}_{A}} \\
& \times \exp \left(\frac{\mathscr{E}}{2 \Re}\left(\frac{1}{T_{b}}-\frac{1}{T_{a}}\right)\right),
\end{aligned}
$$

where $T_{a}$ is the adiabatic flame temperature, $T_{b}$ a burned gas temperature of the steady spherical flame, $T_{u}$ an unburned gas temperature, $U_{a}$ an adiabatic laminar burning velocity, $\rho$ the density, $C_{p}$ the constant pressure specific heat, $k$ the thermal conductivity, $\mathrm{Le}_{A}$ the Lewis number of the deficient reactant $A, \mathscr{E}$ the activation energy, and $\Re$ the universal gas constant. This radius corresponds to the marginal state separating the growth and collapse of a kernel and provides another basis for an understanding of spark ignition along with the minimum ignition energy. This viewpoint on spherical flame initiation well explains experimental observations in spark ignition such as the existence of a minimum ignition energy, the dependence of a minimum ignition energy upon discharge power and duration, and the extinction of an initially well ignited flame kernel. There are, however, only limited data that show the temporal growth of the flame kernel near the minimum ignition energy for well-defined conditions [29-33]. Further research is needed to explore the effect of gap size, equivalence ratio, and spark power and duration on this radius. This is the prime objective of the present study.

A high-speed photographic study provides data on flame kernel growth that are needed for the development of spark ignition models $[8,9,26$, $29-33,36,37]$. It also helps to explain differences in minimum ignition energy or ignitability observed in some cases [14-16, 18]. In highspeed photographic studies with an inductive type 
ignition source, it has been shown that run-to-run variation in the spark discharge as well as the flame kernel development necessitates continuous photographic recording of a single spark event $[8$, $9,26]$. The temporal distribution of spark energy also needs to be recorded in an experimental flame kernel development study because it influences not only the minimum ignition energy but also the rate of kernel growth $[8,17,19,21,26$, 38-40].

The other important fact in spark ignition is that electrical energy is discharged through the electrodes while the flame kernel develops around the electrodes. The contact of the flame kernel with the electrodes is inevitable and results in energy losses. These losses need to be taken into account in the direct validation of a spark ignition model with experimental data. As shown in Sec. 4 , data collected on the minimum ignition energy can be used to estimate the energy loss during spark ignition and the resulting net energy input contributing to ignition.

The first part of the present experimental study provides data for the validation of a model for flame kernel growth developed in Part II [41]. A high-speed laser schlieren technique is used to record developing spark kernels near the minimum ignition energy. The temporal variations of spark kernel shape and size are obtained from the series of pictures recorded from a single spark event. The current and voltage waveforms of the spark are simultaneously measured with the photographic recordings for the computation of the temporal spark power input. In addition, the minimum ignition energy for various gap sizes is measured for some equivalence ratios in order to set the experimental conditions and to study the energy loss during spark ignition.

The study is divided into two parts: the first part is experimental and the second part deals with an intuitive-analytical modeling of the experimental results [41]. The first part consists of five sections: following this introduction, Sec. 2 describes experimental apparatus and procedures, Sec. 3 discusses experimental results in terms of the critical radius, Sec. 4 deals with the energy loss during spark ignition, and Sec. 5 concludes the study.

\section{EXPERIMENT}

The experimental apparatus and procedures developed in Lim [8], Anderson and Lim [26], and Lim et al. [38] are used in the present study with some modifications. These include electrode material and configuration, optical path, exposure time and magnification. Both electrodes are made of nickel and are $0.5 \mathrm{~mm}$ in diameter, the spark gap is set to $0.5-2.0 \mathrm{~mm}$, an optical path is adjusted to record developing spark kernels for a time of 10-30 ms, and an exposure time of $2 \mu \mathrm{s}$ at 10,000 frames $/ \mathrm{s}$ is used for the kernel image at about 0.25 magnification. The constant volume bomb of stainless steel has an $83 \mathrm{~mm}$ in inner diameter and length. This is substantially larger than the kernel size during the experiment time. A schematic of the high-speed laser schlieren system is shown in Fig. 1. The precise timing for the first and subsequent frames is achieved by switching the laser beam into the test section at predetermined times and durations with an acoustooptic modulator. An inductive type power source (see Lim [8] or Ko [9]) provides the spark with a variable power level and discharge duration. The method developed in Lim [8] and Anderson and Lim [26] is also used to compute the volume and surface area of the spark kernel from digitized information of the kernel boundary and electrodes traced from the projected schlieren picture. Assuming the shape of a kernel to be spherical from the very early stage of spark ignition ( $5 \mu \mathrm{s}$ after spark initiation; time of the first frame) $[8,26]$, the equivalent radius $r_{\text {eq }}$ is calculated as $r_{\text {eq }}=(3 V /(4 \pi))^{1 / 3}$, where $V$ is the volume of the kernel.

A propane-air mixture at atmospheric pressure is used in the experimental study. Minimum ignition energies with an approximate $50 \%$ ignition probability are determined at selected conditions (equivalence ratio $\phi$, gap size $d$, and discharge duration $t_{d}$ ). Measurements of the minimum ignition energy show a dependency on the breakdown voltage (see also Ref. 22). Ignition energies with a breakdown voltage of 4-8 KV are used in finding the minimum ignition energy. Most of the discharges in the present experiment occur within this range. The experiments are carried out with 


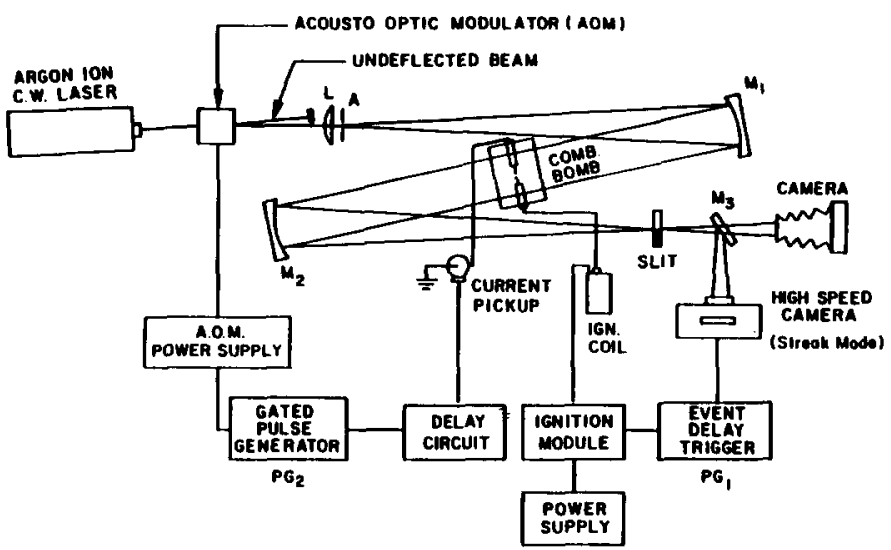

Fig. 1. Schematic of the high-speed laser schlieren system (from Ref. 8).

energy levels slightly above and below this condition (i.e., near the minimum ignition energy) in order to reliably achieve either ignition or nonignition events.

\section{FLAME KERNEL DEVELOPMENT}

Schlieren photographs of a developing flame kernel given in Lim [8], Anderson and Lim [26], and Lim et al. [38] are briefly described below for later convenience. The first frame at $5 \mu \mathrm{s}$ shows an ellipsoidal trace of a weak shock wave as well as a cylindrical kernel. The boundary of the kernel is wavy during the early stage (less than $1 \mathrm{~ms}$ ). This is observed in all the cases studied and is believed to be a result of turbulence created by the blast wave after electrical breakdown of the gap. The kernel boundary becomes smooth in about $1 \mathrm{~ms}$ and the kernel shape becomes nearly spherical thereafter.

The developing flame kernels in the present study appear to be more spherical than those observed by $\operatorname{Lim}$ and colleagues $[8,26,38]$. The small electrode diameter $(D=0.5 \mathrm{~mm})$ and gap size $(d=0.5-2.0 \mathrm{~mm})$ with a relatively lower discharge energy produce a more spherical kernel shape. It is difficult to visually distinguish a difference in kernel growth behavior between ignition and nonignition events up to a considerable time from spark initiation. At later times, which depend on the equivalence ratio, the kernel boundary for the nonignition case begins to fade away with an almost constant radius while the kernel for the ignition case keeps growing.

The equivalent radii of spark kernels in a typical propane-air mixture are plotted versus time in Fig. 2 (see Ko [9] for more experimental results). The experimental conditions for each figure, including the equivalence ratio of the mixture, the gap size, the discharge duration, the average spark energy, and the minimum ignition energy are shown in Table 1. The corresponding spark energy curves are respectively shown in Fig. 3. These data contain both arc (within a short time frame) and glow phases after breakdown. The energy in the arc phase is small (less than $10 \%$ of total) and increases with the total discharge energy (coil primary current). The breakdown energy of about $0.2-1.0 \mathrm{~mJ}$ is not included in Fig. 3.

The critical radius $r_{c}$ for each equivalence ratio, calculated from Eq. 1, is shown in the figures. The flame kernel growth assuming a steady planar adiabatic flame at an arbitrary position is also shown in the figures.

The kernel growth rate for ignition with near minimum energy (Fig. 2) decreases from very large initial values as the radius of the flame kernel approaches the critical radius $r_{c}$. When the kernel radius exceeds the critical radius $r_{c}$, the growth rate accelerates and is expected to eventually approach the adiabatic flame speed. In the 


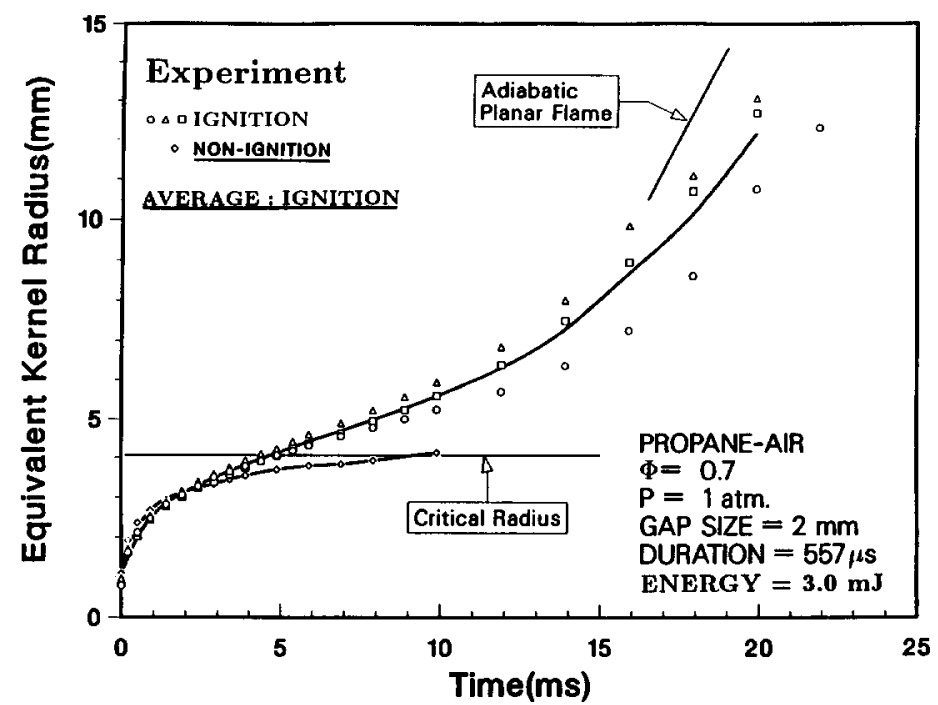

Fig. 2. Temporal growth of spark kernel radius in a propane-air mixture at near minimum ignition energy condition ( $\phi=0.7, d=2 \mathrm{~mm}, t_{d}=557 \mu \mathrm{s}$, and $E_{d}=3.0 \mathrm{~mJ}$ ).

case of nonignition with near minimum energy, the kernel growth rate in the early stage is similar to that for the corresponding ignition case, but the kernel stops growing at the critical radius and subsequently collapses. These observations confirm the existence of the critical radius [33-35, $42]$ and the effect of the critical radius on flame kernel growth [33] with an inductive ignition source. Similar kernel growth is observed in an engine at low engine speed [43]; the rate of kernel growth passes through a minimum at a radius that depends on equivalence ratio and engine speed. This extends the critical radius concept to an engine environment for a limited condition. The observation for a nonignition case confirms the view $[33,34,42]$ that well-ignited flame kernels stop growing if the power input is insufficient to drive the flame kernel beyond the critical radius. The critical radius $r_{c}$ obtained from Eq. 1 compares reasonably well for all cases (see ? Ko [9] for other results).

The run-to-run variation in kernel size (Fig. 2) increases with time for a quiescent mixture with a small-diameter electrode $(D=0.5 \mathrm{~mm})$. It appears that this variation is closely related to the variation in the time for the flame kernel to escape the effect of the critical radius (see Ko [9] for other results). The kernel that escapes the effect of the critical radius earlier has a larger radius later. For a given condition near the minimum ignition energy, the growth beyond the influence of the critical radius is very similar.

TABLE 1

Experimental Conditions

\begin{tabular}{cccrrr}
\hline & $\phi$ & $d(\mathrm{~mm})$ & $t_{d}(\mu \mathrm{s})$ & $E_{d}(\mathrm{~mJ})$ & $E_{m}(\mathrm{~mJ})$ \\
\hline Figs. 2, 4, 5 & 0.7 & 2.0 & 557 & 3.0 & 2.6 \\
Fig. 4 & 0.7 & 1.0 & 565 & 6.2 & 6.0 \\
Fig 4 & 0.7 & 0.5 & 3548 & 38.8 & 35.0 \\
Fig. 5 & 0.8 & 1.0 & 555 & 3.7 & 3.1 \\
Fig. 5 & 0.6 & 2.0 & 3566 & 61.0 & 57.0 \\
\hline
\end{tabular}




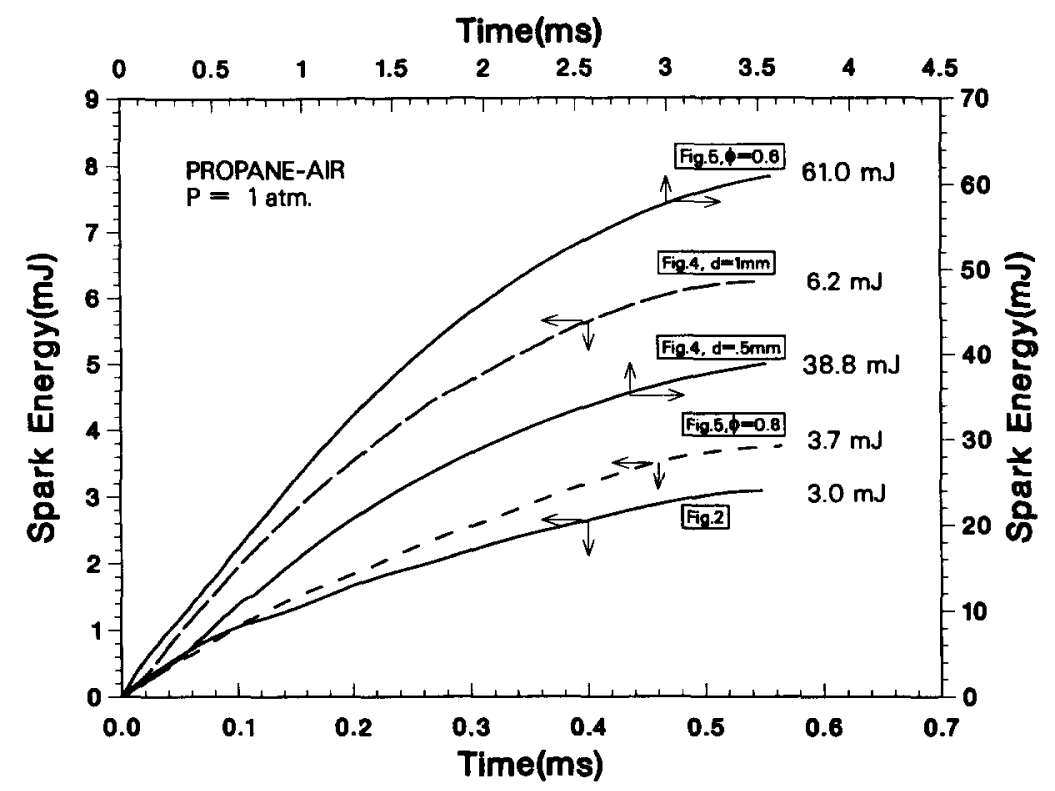

Fig. 3. Temporal distribution of cumulative energy for sparks in Figs. 2, 4, and 5.

The kernel growth when the radius is less than or near the critical radius, however, appears to result in the measured run-to-run variation. This supports the hypothesis that cyclic variations in a homogeneous-charge spark ignition engine originate during the initial period of combustion from the time of spark breakdown to a noticeable departure of cylinder pressure from the compression pressure [44].

The mean profiles for ignition with $\phi=0.7$ are plotted versus time in Fig. 4 to observe the effect of gap size and the related discharge duration on kernel growth near the minimum ignition energy. Data are presented for various gap sizes $(d=0.5-2.0 \mathrm{~mm})$ and related discharge durations $\left(t_{d}=560-3550 \mu \mathrm{s}\right)$. The kernel growths at various conditions near the minimum ignition energy are quite similar for the same equivalence ratio as shown in Fig. 4. Flame initiation, when studied under the theory of the critical radius, requires that $r_{c}$ be sufficiently large when compared to the size of the ignition device [33]. The critical radius for this condition is $4.07 \mathrm{~mm}$ and is shown to remain valid for the 2-mm gap size, which is well within the range of an automotive spark plug. This demonstrates the extension of the theory to actual igniter dimensions.
The theory for the critical radius is also developed for a constant power ignition source [33]. The inductive source used here has variable power and duration. The low power with the $2.0-\mathrm{mm}$ gap is input within $560 \mu \mathrm{s}-\mathrm{a}$ time significantly less than that required to achieve the critical radius ( $\sim 5 \mathrm{~ms}$ ). The higher power case with the $0.5-\mathrm{mm}$ gap is input over a time frame of $3.5 \mathrm{~ms}$, which is of the same order as the time to the critical radius. The data (Fig. 4) show the relative insensitivity of the physics of the critical radius to the ignition power and duration of an inductive ignition source for conditions near the minimum ignition energy.

The mean profiles for successful ignition with $\phi=0.6, \phi=0.7$ (Fig. 2), and $\phi=0.8$ are plotted versus time in Fig. 5 to compare the kernel growth at conditions near the minimum ignition energy for different equivalence ratios. The critical radius increases rapidly with decreasing equivalence ratio, which results in a significant increase in the minimum ignition energy. The kernel approaches the adiabatic flame speed earlier as the equivalence ratio increases. The smaller critical radius and larger combustion-generated energy release and flame speed with increasing equivalence ratio result in an overall 


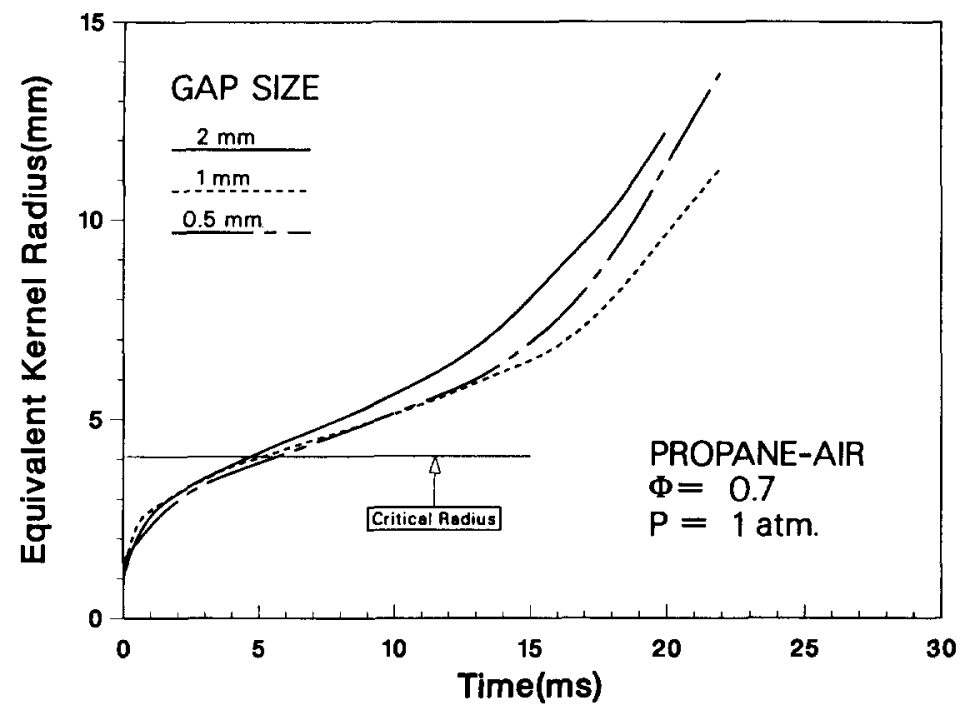

Fig. 4. Effects of gap size on kernel growth at near minimum ignition energy conditions.

faster growth and an earlier approach to the adiabatic flame speed. The larger initial growth exhibited by the leanest equivalence ratio $(\phi=$ $0.6)$ of Fig. 5 is due to the significantly greater spark energy input $(61.0 \mathrm{~mJ}, 3.57 \mathrm{~ms})$ compared to the two other cases $(3.7 \mathrm{~mJ}, 555 \mu \mathrm{s}, 3.0 \mathrm{~mJ}$, $557 \mu \mathrm{s})$. The difficulty in flame initiation as the mixture becomes leaner is clearly shown by the larger critical radius and the longer time for the flame kernel to reach the critical radius even though it initially grows faster because of its much larger energy requirement.

As shown in Fig. 2, the flame speed does not reach the planar adiabatic flame speed at the end

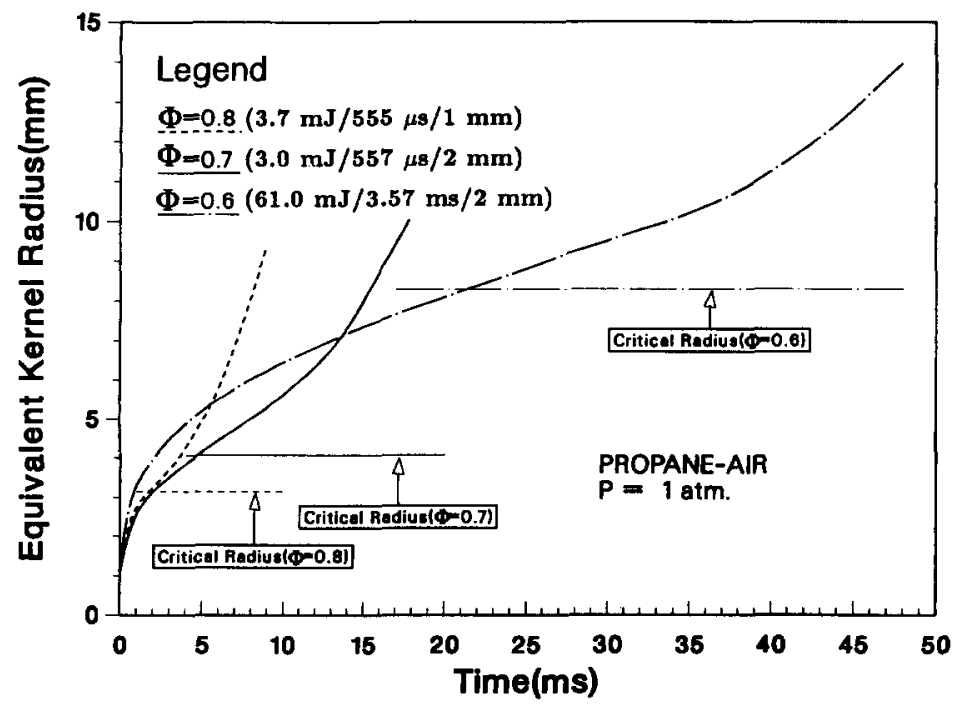

Fig. 5. Effects of equivalence ratio on kernel growth at near minimum ignition energy conditions. 
of each experiment, which is terminated by the optical field of view. The experimental time frame is about $10 \mathrm{~ms}$ for $\phi=0.8,25 \mathrm{~ms}$ for $\phi=0.7$, and $50 \mathrm{~ms}$ for $\phi=0.6$. The flame speed is expected to eventually reach the planar adiabatic flame speed at a later time, since the kernel size is still substantially smaller than the bomb size during the measurement time.

\section{ENERGY LOSS}

During spark ignition of a quiescent mixture, contact of the flame kernel with the electrodes results in energy loss. The major factors affecting energy loss are electrode material and discharge mode (fall energy losses), the properties of the mixture (fall energy losses and quenching loss), electrode temperature and configuration (quenching loss), and discharge duration (time loss). Energy loss due to other heat transfer modes and radical recombination is excluded because it is insignificant with thin electrodes [45] used in the present study. Quantifying these energy losses is essential when using the measured electrical discharge power input for direct validation of the time-dependent ignition model for spark kernel growth in Part II [41]. This is especially important when the gap size is smaller than the quenching distance and when the discharge duration is larger than the optimal duration. In this section, these energy losses are quantified using data on the minimum ignition energy and the fall energy loss and data from spark calorimetry. The actual net spark power for ignition is estimated by reducing the measured total spark power by these losses.

Spark calorimetry is developed to determine the percentage of the spark energy remaining in the noncombustible gas or mixture from measurements of the transient pressure or volume rise in a vessel [46-48]. In spark calorimetry, all the losses are lumped. Quantifying the energy conversion efficiency helps to explain why some ignitors are better than others and may suggest a way to improve the efficiency of ignitors. It is, however, difficult to directly use the data for modifying the electrical power input to an ignition model.
The discharge from a typical inductive ignition source consists of a breakdown, arc, and glow phase $[37,40]$. The fundamental difference between glow and arc discharges is the mechanism of electron supply [49]. The spark energy released in the cathode and anode falls is lost to the electrodes. Only the spark energy discharged within the positive column is available for flame initiation because the fall regions are in close proximity to the electrodes (less than $1 \mu \mathrm{m}$ ). This assumption is supported by experimental evidence that a glow discharge requires a higher total spark energy than an arc discharge [4], but that the difference in the minimum ignition energy between the two discharges vanishes when the spark energy in the positive column is compared [23].

On the basis of the foregoing assumption, the power input to the positive column $\dot{E}_{p}$ is taken as the total spark power minus the power in the fall regions:

$\dot{E}_{p}=I_{s}\left(V_{d}-V_{a, f}-V_{c, f}\right)$,

where $I_{s}$ is the measured secondary current, $V_{d}$ the measured total voltage, $V_{a, f}$ the anode fall voltage, and $V_{c, f}$ the cathode fall voltage. The fall voltages are calculated from Cobine [50]. The arc and glow part of the spark discharge is found on the measured voltage waveform by identifying transition points that are on the order of the cathode fall voltage. These transition regions, when present, occur within a short time frame after the breakdown event. The lower voltage regions adjacent to the transition points are assumed to be arc discharges. A voltage jump on the order of $120 \mathrm{~V}$ is observed between arc and glow regions.

The minimum ignition energy increases with decreasing gap size for gaps less than the quenching distance [2], and with increasing discharge durations larger than the optimal value $[4,10$, 17]. The same trend is found when comparing only the energy input into the positive column $E_{p}$. It implies that the consideration of the fall energy loss alone is inadequate for either the long duration discharge or the smaller gap.

As mentioned above, the fall energy loss can be quantified and dealt with separately. The other 
TABLE 2

Quenching Effect in Data From Figs. 3 and 4 of Kono et al. [17] ${ }^{a, b}$

\begin{tabular}{ccccc}
\hline Gap Size & $3.5 \mathrm{~mm}$ & $2.0 \mathrm{~mm}$ & & $1.0 \mathrm{~mm}$ \\
Duration & $E_{p}(\mathrm{~mJ})$ & $E_{p}(\mathrm{~mJ})$ & & $E_{p}(\mathrm{~mJ})$ \\
$50 \mu \mathrm{s}$ & 0.50 & 0.47 & & 0.60 \\
& & 1.10 & ratio 0.79 & 1.38 \\
$500 \mu \mathrm{s}$ & & & ratio 0.80 & 2.18 \\
$1.0 \mathrm{~ms}$ & & 1.76 & & ratio 0.81 \\
\end{tabular}

${ }^{a}$ Propane-air ( $\phi=0.746$ ), $D=0.3 \mathrm{~mm}$.

${ }^{b} E_{p}=E_{d}-E_{f}, E_{d}$ measured, $E_{f}$ estimated.

remaining losses due to quenching and discharge duration are quantified after removal of the fall energy from the total discharge energy. A method for quantifying the actual net discharge energy used for ignition is unavailable in the literature, though the effect of quenching and discharge duration on minimum ignition energy are experimentally and theoretically well established [2-4, $10,12,13,17,19]$. In the present study, it is shown that data on minimum ignition energy and data from spark calorimetry can be used to account for these two processes.

It is possible to assess the quenching effect by analyzing the data of Kono et al. [17] with the aid of electrode fall data from Ziegler et al. [23]. The results of these analyses are shown in Table 2 , where $E_{p}$ is the estimated positive column energy at each duration and gap size, $E_{d}$ the total measured discharge energy, and $E_{f}$ the estimated energy discharged into the fall regions. The ratios of $E_{p}$ between the 2-mm and 1-mm gap sizes for various discharge durations are approximately the same $(\approx 0.8)$. It is obvious that the energy actually used for ignition with different gap sizes, if other things are equal, must be identical. Therefore, the ratios of $E_{p}$ with different gap sizes to $E_{p}$ at the quenching distance in Table 2, when the discharge durations are identical, shows the quenching effect in terms of the positive column energy $E_{p}$ (i.e., a larger percentage of $E_{p}$ for the 1-mm gap size is lost to the electrodes as a result of quenching than for the 2-mm gap size). It confirms the fact that when the gap size is less than the quenching distance, a certain percentage of $E_{p}$ is lost to the electrodes due to quenching, depending on the equivalence ratio, the gap size and the electrode dimensions. The percentage appears to be nearly independent of the discharge duration up to $1 \mathrm{~ms}$ for a given gap size as shown in Table 2.

For this experiment, the minimum ignition energy for various gap sizes is carefully measured for some equivalence ratios while keeping the discharge duration $t_{d}$ approximately constant for each equivalence ratio. The results are shown in Table $3 . E_{p}$ is assumed to be equal to the energy: contributing to ignition when it does not exhibit any quenching effect, i.e., when $E_{p}$ remains almost constant with increasing gap size. This occurs for a gap size of about $2 \mathrm{~mm}$ at $\phi=0.7$, 0.8 and $3 \mathrm{~mm}$ at $\phi=0.6$. The quenching efficiency in Table $3, \eta_{g}$, is then equal to the ratio between the minimum positive column energy contributing to ignition and $E_{p}$ at each gap size. These data are used in the modeling effort of Part II [41].

The larger minimum ignition energy with increasing discharge duration beyond the optimal value in Table 3 also suggests that energy discharged in the later time of ignition is less efficient. This effect is estimated using experimental data for constant discharge current and power from Fig. 11 of Teets and Sell [48]. Using a conventional spark plug and coil ignition system, the spark energy that remains in air is measured for various discharge durations. The quenching loss is assumed negligible for their data because of the use of a wide gap size $(2 \mathrm{~mm})$ and a lower 
TABLE 3

Quenching Effect in the Experimental Setup ${ }^{a, b}$

\begin{tabular}{ccccccccc}
\hline & \multicolumn{2}{c}{$\phi=0.7$} & \multicolumn{2}{c}{$\phi=0.8$} & \multicolumn{3}{c}{$\phi=0.6$} \\
Duration & \multicolumn{2}{c}{$t_{d} \approx 500 \mu \mathrm{s}$} & \multicolumn{2}{c}{$t_{d} \approx 500 \mu \mathrm{s}$} & \multicolumn{2}{c}{$t_{d} \approx 2100 \mu \mathrm{s}$} & \multicolumn{2}{c}{$t_{d} \approx 2500 \mu \mathrm{s}$} \\
Gap size & $E_{p}$ & $\eta_{g}$ & $E_{p}$ & $\eta_{g}$ & $E_{p}$ & $\eta_{g}$ & $E_{p}$ & $\eta_{g}$ \\
$4 \mathrm{~mm}$ & & & & & 24.5 & & \\
$3 \mathrm{~mm}$ & 1.50 & & & & 24.7 & $\approx 1$ & 28.4 & \\
$2 \mathrm{~mm}$ & 1.55 & $\approx 1$ & 1.03 & & & & 36.3 & 0.78 \\
$1 \mathrm{~mm}$ & 4.25 & 0.35 & 1.29 & 0.80 & & & \\
\hline
\end{tabular}

${ }^{a} E_{p}=E_{d}-E_{f}\left(E_{d}\right.$ measured, $E_{f}$ estimated) $\eta_{g}=E_{p}($ minimum $) / E_{n}$.

${ }^{b} E_{n}=E_{p}$ with $d=n \mathrm{~mm}$ for a given condition.

kernel temperature with air. The resulting factors affecting the efficiency are mainly the fall energies and the discharge duration. For short durations, the energy loss at a very low energy level can be assumed to be only the fall energy loss. Using this approach, when the data of Fig. 11 of Teets and Sell [48] are extrapolated to zero energy level, the efficiency is about $43.5 \%$. This can be assumed as the percentage of spark energy discharged into the positive column from the total energy. Assuming the above, an analysis of the Teets and Sell data is summarized in Table 4. In Table 4, the energy transferred to the positive column $E_{p}$ is calculated by multiplying the total discharge energy by 0.435 . The average discharge efficiency $\eta_{d}^{*}\left(t_{d}\right)$ is defined as $E_{G} / E_{p}$, where $E_{G}$ is the measured spark energy that remains in the gas after the discharge duration $T_{d}$. This efficiency is seen to decrease with increasing discharge duration as the experimental observations suggest.

It is possible to develop an analytical approach for determining the discharge efficiency due to time losses. For constant power,

$\eta_{d}\left(t_{d}\right)=\frac{\int_{0}^{t_{d}} \dot{E}_{p} \eta_{d}(t) d t}{\dot{E}_{p} t_{d}}=\frac{\int_{0}^{t_{d}} \eta_{d}(t) d t}{t_{d}}$,

where $\eta_{d}(t)$ is the instantaneous discharge efficiency at time $t$ and $\eta_{d}\left(t_{d}\right)$ is the average discharge efficiency during the discharge duration $t_{d}$. Assuming $\eta_{d}(t)=\exp \left(-t / t_{0}\right)$, the estimate of $\eta_{d}\left(t_{d}\right)$ compares very well with $\eta_{d}^{*}\left(t_{d}\right)$, as shown in Table 4 when $t_{0}=8 \times 10^{-4} \mathrm{~s}$. With a monotonically decreasing power in time as found with a conventional inductive ignition system (i.e., Fig. 3), the time loss effect is expected to be less than that for constant power in Table 4 because $\eta_{d}(t)$ exponentially cuts off more power with increasing discharge time.

The spark power actually contributing to the ignition process then becomes

$\dot{E}_{G}=\dot{E}_{p} \eta_{g} \eta_{d}(t)$

TABLE 4

Temporal Variation of Discharge Efficiency ${ }^{a, b}$

\begin{tabular}{cccccc}
\hline \multicolumn{3}{c}{ Fig. 11 of Teets and Sell [48] } & \multicolumn{2}{c}{ Estimated } & Eq. 3 \\
\hline$T_{d}(\mathrm{~ms})$ & $E_{d}(\mathrm{~mJ})$ & $E_{G}(\mathrm{~mJ})$ & $E_{p}(\mathrm{~mJ})$ & $\eta_{d}^{*}\left(t_{d}\right)(\%)$ & $\eta_{d}\left(t_{d}\right)(\%)$ \\
0.48 & 12 & 4.08 & 5.22 & 78.2 & 75.2 \\
0.96 & 24 & 6.48 & 10.44 & 62.1 & 58.2 \\
2.0 & 50 & 8.75 & 21.75 & 40.2 & 36.7 \\
3.0 & 75 & 9.38 & 32.63 & 28.8 & 26.0 \\
\hline
\end{tabular}

${ }^{a} E_{p}=E_{d} \times 0.435, \eta_{d}^{*}\left(t_{d}\right)+E_{G} / E_{p}, E_{d}=$ total measured energy.

${ }^{b} E_{G}=$ measured gas energy, $t_{0}=8 \times 10^{-4}(\mathrm{~ms})$ for $\mathrm{Eq} .3$. 
where $\dot{E}_{G}$ is the actual power input contributing to ignition. The efficiency $\eta_{g}$ accounts for the quenching effect and $\eta_{d}(t)$ for time loss effects after removal of the fall losses (Eq. 2). Note that this approach is more comprehensive than that in the literature where the efficiency is found from $E_{G}=\eta \times E_{d}[5,46-48]$. The additional complexity is necessary because ignition is strongly dependent upon the power input (not just total energy), as is shown in Refs. 5, 9, 24, 25, 33, 34,37 , and 39 .

\section{CONCLUSIONS}

The developing spark kernel from an inductive ignition source in a lean propane-air mixture is photographed with a high-speed laser schlieren system. Current and voltage waveforms of the spark are simultaneously measured with photographic recordings for computation of the temporal spark power input. The temporal growth of the measured equivalent kernel radii is investigated at conditions near the minimum ignition energy for some equivalence ratios $(\phi=$ $0.6-0.8)$, gap sizes $(d=0.5-2.0 \mathrm{~mm})$, and discharge durations $\left(t_{d}=550 \mu \mathrm{s} \sim 3.5 \mathrm{~ms}\right)$. The minimum ignition energy for various gap sizes is measured for some equivalence ratios to study the energy loss during spark ignition. These experimental data are used in Part II [41] to develop a model for kernel growth. The major findings of the study are as follows:

(1) The effect of the critical radius on kernel growth is determined under various conditions for the inductive type ignition source. For ignition near the minimum ignition energy, the flame speed decreases from a very large value at early stages as the radius of the flame kernel becomes closer to the critical radius $r_{c}$. As the flame kernel radius becomes larger than the critical radius $r_{c}$, the speed increases and eventually approaches the planar adiabatic flame speed. For nonignition with a near minimum ignition energy, the kernel growth in the early stage is similar to that for the corresponding ignition case, but the kernel stops growing at a radius smaller than the critical radius $r_{c}$ and eventually extinguishes.

(2) The explicit expression for the critical ra- dius $r_{c}$ from Champion et al. [33] (Eq. 1) predicts the critical radius reasonably well for the conditions examined.

(3) The influence of the critical radius on kernel growth is valid when discharge durations are shorter than the time scale for the kernel to reach the critical radius, and when the critical radius $r_{c}$ is at least a factor of $\mathbf{2}$ or larger than the characteristic dimensions of the ignition device such as the gap size and the electrode diameter.

(4) The temporal growth of kernels near the minimum ignition energy and with the same equivalence ratio at different gaps sizes and vastly different measured ignition energies $(3.0-39 \mathrm{~mJ})$ are found to be very similar and in experimental scatter.

(5) The kernel not only grows faster in size but also starts to approach the planar adiabatic flame speed earlier as the equivalence ratio increases.

(6) The flame speed does not reach the steady planar adiabatic flame speed at the end of each photographic recording, which is about $10 \mathrm{~ms}$ for $\phi=0.8,20 \mathrm{~ms}$ for $\phi=0.7$, and $50 \mathrm{~ms}$ for $\phi=0.6$ and is limited by the field of view of the optical system. However, it is expected to eventually approach the planar adiabatic flame speed before reaching the walls of the combustion bomb.

(7) The actual net spark power for ignition can be estimated by adjusting the measured total spark power for the effects of electrode fall losses, quenching, and time losses. These effects can be estimated from available data.

\section{REFERENCES}

1. Quader, A. A., SAE Paper 760760, 1976.

2. Lewis, B. L., and von Elbe, G., Combustion, Flames, and Explosions of Gases, Academic, New York, 1961.

3. Ballal, D. R., and Lefebvre, A. H., Proc. R. Soc. Lond. A 357:163-181 (1977).

4. Swett, C. C. Jr., NACA T.R. 1287, 799-816, 1956.

5. Maly, R., Fuel Economy in Road Vehicles Powdered by Spark Ignition Engines (J. C. Hilliard and G. S. Springer, Eds.), Plenum, New York, 1984, pp. 91-144.

6. Kalghatgi, G. T., SAE Paper 870163, 1987.

7. Heywood, J. B., Internal Combustion Engine Fundamentals, McGraw-Hill, New York, 1988.

8. Lim, M. T., Ph.D. thesis, The University of Michigan, Ann Arbor, 1985. 
9. Ko, Y., Ph.D. thesis, The University of Michigan, Ann Arbor, 1989.

10. Ballal, D. R., and Lefebvre, A. H., Combust. Flame 24:99- 108 (1975).

11. Ballal, D. R., and Lefebvre, A. H., Fifteenth Symposium (International) on Combustion, The Combustion Institute, Pittsburgh, 1974, pp. 1473-1481.

12. Calcote, H. F., Gregory, C. A., Jr., Barnett, C. M., and Glimer, R. B., Ind. Eng. Chem. 44:2656-2662 (1952).

13. Rose, H. E., and Priede, T., Seventh Symposium (International) on Combustion, Butterworths, London, 1959, pp. 436-445.

14. Hattori, T., Goto, K., and Ohigashi, S., Proc. Inst. Mech. Eng. (Lond.) 153-164 (1979).

15. Kono, M., Kumagai, S., and Sakai, T., Combust. Flame 27:85-98 (1976).

16. Kono, M., and Hatori, K., Eighth International Conference on Gas Discharges and Their Applications, Pergamon, London, 1985, pp. 500-503.

17. Kono, M., Kumagai, S., and Sakai, T., Sixteenth Symposium (International) on Combustion, The Combustion Institute, Pittsburgh, 1977, pp. 757-766.

18. Kono, M., Hatori, K., and Iinuma, K., Twenty-First Symposium (International) on Combustion, The Combustion Institute, Pittsburgh, 1984, pp. 133-140.

19. de Soete, G. G., Proc. Inst. Mech. Eng. Part C 93-100 (1983).

20. de Soete, G. G., Thirteenth Symposium (International) on Combustion, The Combustion Institute, Pittsburgh, 1971, pp. 735-743.

21. Steiner, J. C., and Mirsky, W., SAE Paper 660346, 1966.

22. Tromans, P. S., and O'Connor, S. J., Prog. Astronaut. Aeronaut. 95:421-432 (1985).

23. Ziegler, G. F. W., Wagner, E. P., and Maly, R. R., Twentieth Symposium (International) on Combustion, The Combustion Institute, Pittsburgh, 1984, pp. 1817-1824.

24. Karpov, V. N., Malov, V. V., and Severin, E. S., Combust. Explos. Shock Waves 22:707-712 (1987).

25. Malov, V. V., Eighth International Conference on Gas Discharges and Their Applications, Pergamon, London, 1985, pp. 507-510.

26. Anderson, R. W., and Lim, M. T., Eighth International Conference on Gas Discharges and Their Applications, Pergamon, London, 1985, pp. 511-514.

27. Tromans, P. S., and Furzeland, R. M., Tenth International Colloquium on (Gas) Dynamics of Explosions and Reactive Systems, 1985.
28. Sloane, T. M., Tenth International Colloquium on (Gas) Dynamics of Explosions and Reactive Systems, 1985.

29. Arnold, J. S., and Sherburne, R. K., Fourth Symposium on Combustion, Williams Wilkins, Baltimore, 1953, pp. 139-143.

30. Lintin, D. R., and Wooding, E. R., Br. J. Appl. Phys. 10:159-166 (1959).

31. Akindele, O. O., Bradley, D., Mak, P. W., and McMahon, M., Combust. Flame 47:129-155 (1982).

32. Olsen, H. L., Gayhart, E. L., and Edmonson, R. B., Fourth Symposium (International) on Combustion, Williams \& Wilkins, Baltimore, 1953, pp. 144-148.

33. Champion, B., Deshaies, B., Joulin, G., and Kinoshita, K., Combust. Flame 65:319-337 (1986).

34. Deshaies, B., and Joulin, G., Combust. Sci. Technol. 37:99-116 (1984).

35. Zeldovich, Ya. B., Barenblatt, G. I., Librovich, B. V., and Makhviladze, G. M., The Mathematical Theory of Combustion and Explosions, Nauka, Moscow, 1980.

36. Litchfield, E. L., Combust. Flame 5:235-241, (1961).

37. Maly, R., and Vogel, M., Seventeenth Symposium (International) on Combustion, The Combustion Institute, Pittsburgh, 1979, pp. 821-831.

38. Lim, M. T., Anderson, R. W., and Arpaci, V. S., Combust. Flame 69:303-316 (1987).

39. Adelman, H. G., Eighteenth Symposium (International) on Combustion. The Combustion Institute, Pittsburgh, 1981, pp. 1333-1342.

40. Albrecht, H., Bloss, W. H., Herden, W., Maly, R., Saggau, B., and Wagner, E., SAE Paper 770853, 1977.

41. Ko, Y., Arpaci, V. S., and Anderson, R. W., Combust. Flame 83:88-105 (1991).

42. Joulin, G., Combust. Sci. Technol. 43:99-113 (1985).

43. Baritaud, T. A., SAE Paper 872152, 1987.

44. Young, M. B., SAE Paper 810020, 1981.

45. Ko, Y., and Anderson, R. W., SAE Paper 892083, 1989.

46. Roth, W., Guest, P. G., von Elbe, G., and Lewis, B., J. Chem. Phys. 19:1530-1535 (1951).

47. Saggau, B., Arch. Elektrotech. 64:229-235 (1981).

48. Teets, R. E., and Sell, J. A., SAE Paper 880204, 1988.

49. von Engel, A., Ionized Gases, Claredon, Oxford, 1955.

50. Cobine, J. D., Gaseous Conductors, Dover, New York, 1958.

Received 22 August 1989; revised 13 March 1990 